\title{
Tecnología lítica del Holoceno tardío en la estepa fueguina. Actividades de talla y decisiones tecnológicas en Avilés 3
}

\section{(1) Sabrina Labrone*}

Fecha de defensa: 10 de abril de 2019

Director: Dr. Fernando Santiago

Co-directora: Dra. Mariana Carballido

Jurados: Lic. Teresa Civalero y Dra. Mariana

Sacchi

\section{Introducción: área de estudio y problemática}

Las investigaciones arqueológicas en el norte de la Isla Grande de Tierra del Fuego, que comenzaron a desarrollarse a fines del siglo XIX y se volvieron más sistemáticas hacia la década de 1970 (Oría et al., 2017), cubrieron y cubren distintas temáticas y lapsos a través de un abanico diverso de estudios: análisis distribucionales, zooarqueología, isótopos estables, geoarqueología, tafonomía, tecnología lítica y ósea. En esta tesis se buscó aportar nuevos datos, a partir de la tecnología lítica, acerca de los grupos cazadoresrecolectores que ocuparon este sector septentrional de la isla, considerando el registro del sitio Avilés 3.

El presente trabajo se focaliza sobre el área que corresponde al territorio demarcado por el río Chico y Avilés hacia el norte, el río Grande por el sur, el límite internacional con Chile por el oeste y el océano Atlántico por el este, en un ambiente de estepa. Específicamente Avilés 3 se localiza al sur oeste de la confluencia del río Avilés con el río Chico (Figura 1). En este sitio se registraron materiales líticos y óseos en superficie, al interior de una hoyada de deflación (Santiago, 2013). Si bien hasta el momento no se cuenta con una cronología absoluta, algunos autores, en base a evidencias contextuales y comparación con otro sitio del área, asignaron una cronología tentativa para estos materiales correspondiente al Holoceno tardío (Santiago, 2013; Turnes, 2014).

Previo al desarrollo de esta tesis, se planteó a manera de hipótesis que Avilés 3 funcionó como un taller lítico. Este planteo surgió a partir de ciertas evidencias tales como la concentración de guijarros cercanos al sitio y en el entorno inmediato y a la mayor cantidad de evidencia de talla lítica (desechos de talla) en detrimento de los instrumentos, así como la ausencia de restos de fauna con

\footnotetext{
* Laboratorio de Geomorfología y Cuaternario, Centro Austral de Investigaciones Científicas (CADIC), CONICET / Instituto de Cultura, Sociedad y Estado, Universidad Nacional de Tierra del Fuego (UNTdF). B. Houssay 200 (CP V9410CAB) Ushuaia, Tierra del Fuego, Argentina. E-mail: slabrone@gmail.com; slabrone@untdf.edu.ar
}

claras marcas antrópicas (Santiago, 2013; Turnes, 2014). Puntualmente, los análisis detallados sobre el registro lítico se habían concentrado sobre las raederas, de los cuales se obtuvo información sobre las materias primas utilizadas para la confección de estos artefactos, las técnicas de talla empleadas y la habilidad técnica involucrada en su manufactura (Turnes, 2014). Sin embargo, existía un vacío de información sobre las etapas de la producción lítica reflejadas en el sitio y las características del proceso de manufactura en general (como las técnicas de talla utilizadas, las formas base buscadas, energía invertida, entre otras). De acuerdo con lo expuesto, en esta tesis se retomó el estudio de los instrumentos, iniciado en Turnes (2014), y se incorporó el análisis de los núcleos y desechos de talla. Principalmente estos últimos son los que permanecen en el lugar de producción (Ahler, 1987), por lo que son los más adecuados para identificar y precisar actividades realizadas en el sitio. No obstante, las tres clases artefactuales constituyen vías de análisis complementarias.

\section{Objetivos de investigación}

A partir de la problemática mencionada en el apartado anterior, se planteó como fin último aportar al conocimiento sobre las actividades tecnológicas desarrolladas en el sitio Avilés 3, las decisiones que los grupos humanos tomaron sobre la tecnología lítica, en particular, las estrategias que implementaron en la manufactura, uso y descarte de los instrumentos líticos, y precisar el rol que cumplió este sitio en el sistema de asentamiento durante el Holoceno tardío. Para alcanzar estos propósitos se formularon los siguientes objetivos particulares: caracterizar las actividades tecnológicas llevadas a cabo en Avilés 3, examinar el aprovechamiento diferencial de las materias primas locales en relación a sus calidades para la talla, inferir aspectos de las estrategias tecnológicas desarrolladas por los grupos que habitaron este sitio y, por último, discutir e incorporar los datos obtenidos con la información sobre los otros sitios de la región de estudio correspondientes al Holoceno tardío.

\section{Metodología y marco teórico}

La información pertinente para alcanzar estos objetivos se obtuvo del análisis tecnomorfológico de la totalidad del conjunto artefactual lítico recuperado de Avilés 3 (777 artefactos) que fue clasificado en cuatro categorías: 


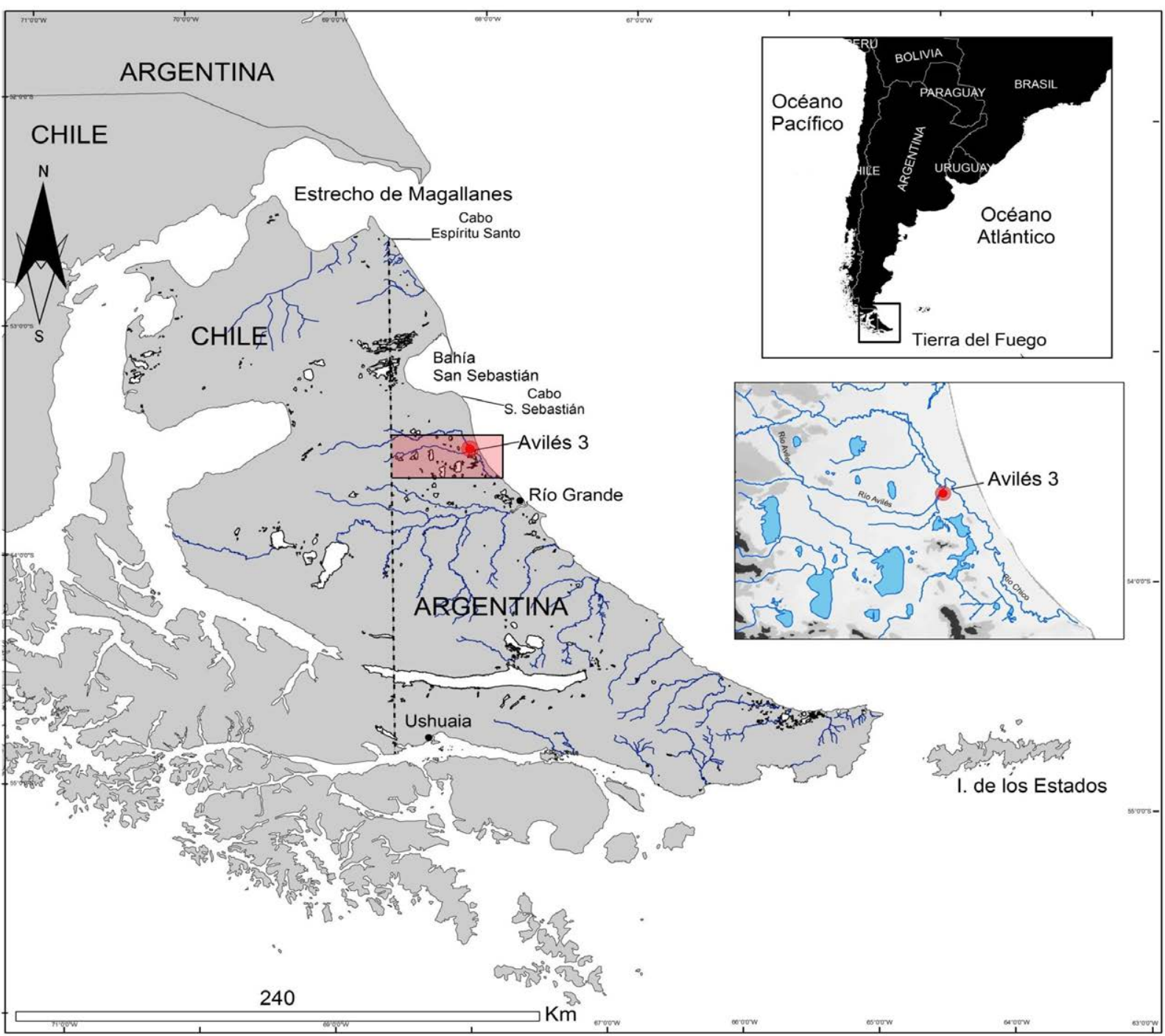

Figura 1. Ubicación del área de estudio (recuadro rojo) y del sitio Avilés 3 (punto rojo).

\begin{tabular}{|l|c|c|}
\hline Clase tipológica & N & \% \\
\hline Núcleos & 16 & 2,05 \\
Desechos de talla & 737 & 94,85 \\
Artefactos formatizados & 20 & 2,6 \\
FNCRC* & 4 & 0,5 \\
\hline Total & 777 & $\mathbf{1 0 0}$ \\
\hline
\end{tabular}

Tabla 1. Clases tipológicas registradas en Avilés 3. "FNCRC: filos naturales con rastros complementarios.

núcleos, desechos de talla, artefactos formatizados y filos naturales con rastros complementarios (Tabla 1). Para su análisis se siguieron principalmente los lineamientos de Aschero (1975, 1983), se utilizó la estadística descriptiva para el análisis de los resultadosy se retomaron los datos ya sistematizados por investigaciones previas para la comparación con los otros sitios del área. La información obtenida se interpretó a partir de los supuestos teóricos del enfoque de la organización tecnológica (sensu Nelson, 1991), para evaluar las estrategias tecnológicas desarrolladas por los grupos humanos en la producción, uso y descarte de los instrumentos líticos. Particularmente en este trabajo, se focalizó sobre algunos de los factores que influyen en la toma de decisiones: la disponibilidad y distribución de las materias primas, la movilidad del grupo, y la naturaleza del recurso a explotar.

\section{Resultados y conclusiones}

Las actividades tecnológicas representadas en el sitio indican una representación diferencial de las etapas involucradas en la manufactura, interpretación que surge de la información obtenida de los núcleos, tamaños y tipos de lascas en los desechos y tamaño de los instrumentos y estado del filo de los mismos. Se registraron principalmente evidencias relacionadas a actividades de extracción de 
formas base y formatización de instrumentos y en menor medida el desbaste inicial de las masas líticas. Los datos no fueron suficientes para discutir la presencia de tareas de formatización final de instrumentos y/o la reactivación de sus filos. Todos los instrumentos identificados se relacionan con tareas de procesamiento de recursos, lo que se ve reforzado por la ausencia en el sitio de artefactos para la caza, como puntas de proyectil o bolas de boleadora.

Para la manufactura de los artefactos líticos, los datos de las materias primas muestran un predominio en el uso de rocas locales, de las cuales la calcedonia ${ }^{1}$ evidencia una explotación diferencial, con una intensidad de uso mayor respecto de las otras materias primas. A su vez, la evidencia en el registro lítico de artefactos confeccionados en rocas de buena y muy buena calidad para la talla, permite sugerir una selección muy cuidadosa de las materias primas. No obstante, se observó que los grupos humanos no buscaron y/o no necesitaron maximizar el rendimiento de estas rocas, lo que indica un comportamiento no económico o de derroche, que se condice con la abundancia y disponibilidad de las materias primas, las cuales se encontraban cercanas al sitio e inmediatamente disponibles (Civalero y Franco, 2003; Salemme, Oría, Turnes y Santiago, 2014; Turnes et al., 2016).

Como último punto, el análisis tecnomorfológico brindó evidencias para postular que los grupos cazadoresrecolectores priorizaron una estrategia tendiente a la expeditividad en el proceso de manufactura, uso y descarte de los instrumentos líticos. Las evidencias del desbaste inicial de los núcleos, las formas no estandarizadas de estos, la gran frecuencia de artefactos con remanentes de corteza, la falta de estandarización en los tipos y tamaños de los artefactos y ejemplares que requirieron de una baja inversión de energía (instrumentos unifaciales, con retoque marginal, filos naturales con rastros complementarios, sumado a escasas o nulas evidencias de talla bifacial) indican la elección de esta estrategia. Esta tendencia también se registró en una escala mayor, al relacionar los datos obtenidos de Avilés 3 con los de los otros sitios del área (Oría, 2012; Santiago, 2013; Santiago y Oría, 2007), no obstante, la información disponible para el norte de la isla sugiere que los humanos alternaron y combinaron estrategias tecnológicas expeditivas y conservadas en la producción, uso y descarte de sus instrumentos líticos (Borrazzo, 2010; Huidobro, 2010; Massone, 2004; Oría,

${ }^{1}$ De acuerdo con la necesidad de trabajar con terminologías comunes para la denominación de las materias primas (Alberti y Fernández, 2015), aclaramos que aquellas clasificadas en este trabajo como calcedonias refieren a rocas de muy buena y buena calidad para la talla, de textura afanítica (grano fino) y fractura concoide, equivalentes a las rocas silicificadas descriptas en Borrazzo (2010, p. 173). Se considera necesario utilizar categorías de clasificación que puedan ser reconocidas a ojo desnudo (como las descriptas en Borrazzo, 2010) y resignar la determinación específica en los casos en que se cuente con cortes delgados o su asignación a ojo desnudo sea muy evidente. Estas consideraciones se tomarán en cuenta para futuros trabajos.
2012; Prieto, Calás y Torres, 2007; Santiago, 2013; Santiago y Oría, 2007).

A partir de la información expuesta, se concluye que Avilés 3 funcionó como un sitio de actividades específicas, relacionadas con la talla lítica, en la cual los grupos humanos llevaron a cabo principalmente tareas de extracción de formas-base y formatización de instrumentos. A su vez, los cazadores-recolectores aprovecharon la oferta local de materias primas, explotando en mayor medida aquellas rocas de mejor calidad para la talla. Además, el conjunto lítico de este sitio mostró una tendencia hacia la aplicación de una estrategia expeditiva en la manufactura, uso y descarte de los instrumentos líticos, tendencia que, como se mencionó, se mantiene para el resto del área.

El desarrollo de este trabajo permitió ampliar el conocimiento sobre la tecnología lítica de Avilés 3, aportando un antecedente más de investigación en la arqueología del norte de Tierra del Fuego.

\section{Referencias citadas}

»Ahler, S. A. (1987). Mass analysis of flaking debris: studying the forest rather than the tree. Archeological Papers of the American Anthropological Association, 1(1), 85-118.

» Alberti, J. y Fernández, M. V. (2015). Propuesta clasificatoria para las materias primas líticas en Patagonia (Argentina). Arqueología, 21(2), 211-235

» Aschero, C. A. (1975). Ensayo para una clasificación morfológica de los artefactos líticos aplicada aestudios tipológicos comparativos. Informe al CONICET. Manuscrito inédito.

» Aschero, C. A (1983). Ensayo para una clasificación morfológica de los artefactos líticos aplicada aestudios tipológicos comparativos. Informe al CONICET. Manuscrito inédito.

» Borrazzo, K. (2010). Arqueología de los esteparios. Tecnología y tafonomía lítica en el norte de Tierra del Fuego, Argentina. (Tesis Doctoral inédita), Universidad de Buenos Aires, Argentina.

»Civalero, T. yFranco, N. (2003). Early human occupations in Western Santa Cruz province, Southernmost South America. Quaternary International, 109, 77-86.

» Huidobro, C. (2010). Métodos de reducción bifacial del norte de Tierra del Fuego durante el Holoceno medio y tardío. (Tesis de Licenciatura inédita), Universidad Nacional de Chile, Chile.

» Massone, M. (2004). Los cazadores después del hielo. Santiago de Chile: Centro de Investigaciones Diego Barros Arana. 
» Nelson, M. (1991). The study of technological organization. En M. Schiffer (Ed.), Archaeological method and theory (Volumen3) (pp. 57-100). Tucson: University of Arizona Press.

"Oría, J. (2012). Patrones de movilidad pre-europeos en el norte de Tierra del Fuego. Una aproximación geoarqueológica. (Tesis Doctoral inédita), Universidad Nacional de La Plata, Argentina.

» Oría, J., Vázquez, M., Borrero, L., Borrazzo, B. y Barberena, R. (2017). Arqueología del Holoceno tardío en la costa Atlántica fueguina. En M. Vázquez, D. Elkin y J. Oría (Eds.), Patrimonio a orillas del mar, arqueología del litoral atlántico de Tierra del Fuego (pp. 87-102). Ushuaia: Editora Cultural Tierra del Fuego.

"Prieto, A., Calás, E.y Torres, J. (2007). El sitio arqueológico Myren 2, Tierra del Fuego, Chile. Magallania, 35(2), 89103.

» Salemme, M., Oría, J., Turnes, L. y Santiago, F. (2014). Caracterización tecnomorfológica de dos conjuntos líticos del Holoceno medio en la estepa fueguina:
La Arcillosa 2 y Río Chico 1. Revista del Museo de Antropología, 7(1), 39-54.

"Santiago, F. (2013). La ocupación humana del norte de Tierra del Fuego durante el Holoceno medio y tardío. Su vinculación con el paisaje. Ushuaia: Editora Cultural Tierra del Fuego.

»Santiago, F. y Oría, J. (2007). Lo que el viento no se llevó. Análisis de sitios de superficie en la estepa fueguina. Magallania, 35(2), 121-132.

"Turnes, L. (2014). Retouched artifacts production in three hunter-gatherer contexts from Tierra del Fuego (southernmost South America, Argentina): Avilés 1, Avilés 3 and Herradura1. Quaternary International, 375, $113-123$.

" Turnes, L., Quiroga, D., Santiago, F., Negre Pérez, J. y González Guillot, M. (2016). Primera aproximación al estudio de fuentes secundarias de materias primas (área intercuencas río Avilés - río Chico, Tierra del Fuego, Argentina). Intersecciones en Antropología, 4, 518. 